A

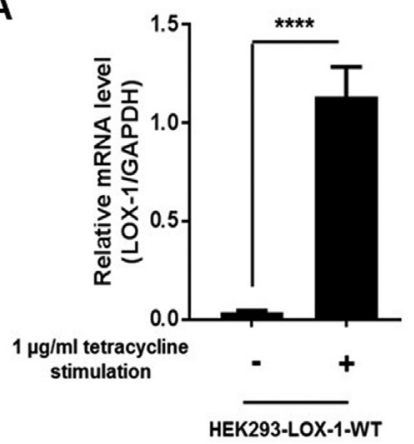

B

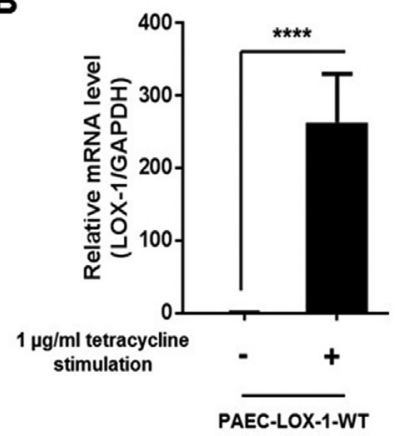

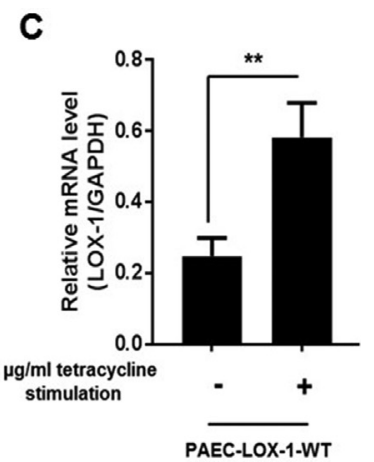

Abstract 163 Figure 1 Relative mRNA levels of: A) Human LOX-1 in HEKs, B) Human LOX-1 in PAECs and C) Porcine LOX-1 in PAECs

A
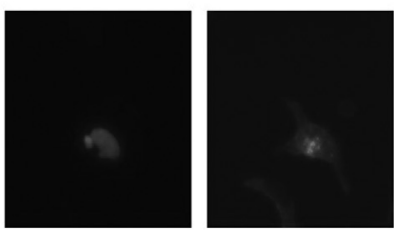

B

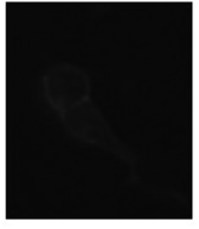

0

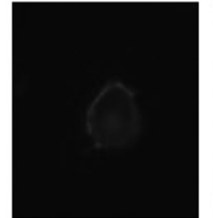

5
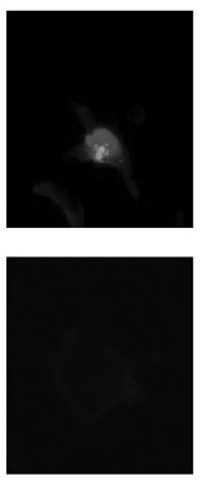

50

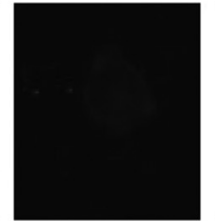

500

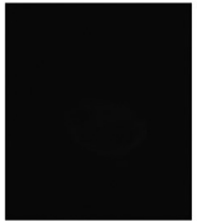

5000

Abstract 163 Figure 2 Immunofluorescence Microscopy of HEK293T after induction with 1microgram/ml Tetracycline. A) Blue (DAPI) staining of nucleus and green (anti-FLAG) staining of FLAG-tagged LOX-1 B) Affimer effects on oxLDL (red) uptake.

(DiI)-labelled oxLDL (10 microgram/ml) for $30 \mathrm{~min}$. Cells were fixed and imaged to characterise the uptake of OxLDL. Blocking experiments using 5 different affimers, was carried out by adding affimer prior to incubation with DiI-oxLDL. Effects on oxLDL binding and cellular accumulation was evaluated using fluorescence microscopy.

Results The levels of LOX-1-FLAG mRNA were significantly raised $(p<0.001)$ in both cell types in response to tetracycline. Porcine endothelial cells also had significant levels of endogenous native porcine LOX-1(Figure 1). The presence of human LOX-1-FLAG protein was confirmed by immunofluorescence microscopy (Figure 2a). Incubation with oxLDL demonstrated tetracycline-dependent labelled lipid particle uptake in HEK293T LOX-1-FLAG expressing cells. In contrast, oxLDL uptake was not significantly raised compared to uninduced baseline in PAEC LOX-1-FLAG expressing cells. Incubation with LOX-1 binding affimers led to a decrease in oxLDL uptake in both cell lines after induction of LOX-1-FLAG expression (Figure 2b).

Conclusion We have successfully constructed two mammalian cell lines with a stable, inducible LOX-1 expression system. This has allowed us to demonstrate LOX-1-dependent oxLDL uptake. However, such expression is dependent on negligible expression of other scavenger receptors that mediate oxLDL binding and/or uptake. The use of LOX-1-specific affimers show that oxLDL binding and/or uptake is significantly decreased in both cell lines. Future experiments aim to determine the effects of such LOX-1-specific affimers in primary vascular cells and animal models in the context of atherosclerosis and vascular disease.

\section{ABSTRACT WITHDRAWN}

\section{HUMAN ATHEROSCLEROSIS IS CHARACTERISED BY OXIDATIVE DNA DAMAGE DUE TO DEFECTIVE BASE EXCISION REPAIR}

Aarti Shah*, Kelly Gray, Nichola Figg, Martin Bennett. University of Cambridge

\subsection{6/heartjnl-2017-311726.163}

Rationale Human atherosclerotic plaques show extensive oxidative DNA damage in vascular smooth muscle cells (VSMCs) and macrophages, including accumulation of 8-oxo-7,8-dihydroguanine (8-oxoG), the most abundant DNA base lesion on oxidative exposure. 8 -oxoG is repaired by base excision repair (BER) mediated by DNA glycosylases, including the specific non-redundant 8-oxoguanine DNA glycosylase-1 (OGG1). OGG1 activity is regulated by acetylation through the p300 acetyltransferase. However, the role of oxidative damage in VSMC function and the regulation of OGG1 in atherosclerosis are unknown.

Methodology We stably expressed OGG1 or the ${ }^{\mathrm{K} 338 / \mathrm{K} 341} \mathrm{OGG} 1$ acetylation mutant in rat VSMCs in vitro, and treated cells with oxidative stress. The functional effects of OGG1 in vivo were 
studied using global OGG1 ${ }^{-/-}$mice and novel VSMC-specific transgenic mice lines expressing OGG1 or ${ }^{\mathrm{K} 338 / \mathrm{K} 341} \mathrm{OGG} 1$. BER activity was measured via incision cleavage of an 8-oxoG-containing oligonucleotide, 8-oxoG levels by ELISA, and protein binding/ interaction by immunoprecipitation and western blotting. OGG1, acetyl OGG1, p300, and 8-oxoG expression were assayed in mouse arteries and human plaques by immunohistochemistry.

Results Nuclear BER activity was defective in atherosclerotic plaque VSMCs, which correlated with a concomitant decrease in acetylation of OGG1 and OGG1 protein expression. In vitro knockout of OGG1 by CRISPR/CAS9 indicated that OGG1 is the major BER DNA glycosylase in VSMCs. Consistently, OGG1 expression increased BER activity and reduced 8-oxoG levels in vitro and in vivo, features not seen with the K338/K341 OGG1 acetylation mutant, indicating that acetylation regulates OGG1 activity in human VSMCs.

OGG1 knockout cells displayed increased sensitivity to DNA breaks induced by oxidant stress and increased apoptosis compared to control cells, measured by comet assay and Annexin-V/propidium iodide FACS staining respectively. This effect was blunted by overexpression of OGG1 but not K338/ ${ }^{\mathrm{K} 341}$ OGG1, suggesting a protective effect of OGG1 against DNA damage, and that the acetylation status of OGG1 is crucial for its function. Immunoprecipitation studies showed that p300 binds to OGG1, and this interaction was reduced under oxidative stress. Furthermore, levels of p300 were decreased in human plaque VSMCs and in response to oxidative stress, suggesting that ROS-induced regulation of OGG1 acetylation could be due to ROS-induced decreased in p300 expression and regulation of its interaction with OGG1.

Conclusions In conclusion, nuclear BER activity is reduced in human atherosclerotic plaque VSMCs, associated with reduced OGG1 acetylation and activity, potentially mediated by reduced p300. Mice with decreased and increased OGG1 will be used to study whether oxidative damage promotes plaque progression in atherosclerosis.

\section{CHANGES IN E-C COUPLING PROTEINS AND TRANSVERSE AND AXIAL TUBULAR STRUCTURES IN GUINEA PIG VENTRICULAR MUSCLE DURING PRE- AND POST-NATAL DEVELOPMENT TO ADULTHOOD}

Hanan Kashbour*, Michael Taggart, Kathryne White. Newcastle university

10.1136/heartjnl-2017-311726.164

Transverse tubules (TT), plasmalemma invaginations perpendicular to the long axis of the cardiomyocyte, facilitate rapid action potential transmission to the cell interior and efficient cardiac excitation-contraction coupling (ECC). Tubule alteration and changes in expression of proteins important for normal ECC have been noted in heart disease. The aim of this study is to explore the changes of selected ECC-related protein expressions, and transverse tubular structures, from preand post-natal cardiac development to adulthood in guinea pig ventricular muscle. Hearts were collected from guinea pigs at developmental stages: fetal (between gestation days (G) 55-68; term=G67-68); neonatal week one (NW1), two (NW2) and three (NW3); and adult. Hearts were flushed with a cold cardiologic solution and (i) left ventricles (LV) frozen for subsequent protein analysis by western blotting or (ii) retrogradely perfused with fixative and LV processed for ultrastructural examination by serial block face-scanning electron microscopy. Values are mean \pm SEM, compared by one-way ANOVA and Bonferroni posthoc test $(p<0.05)$. Expression of $\hat{\mathrm{I}}^{2}$ adrenoceptor, TT marker, increased in adults $(0.87$ \pm 0.14 fold relative to positive control) and neonates NW2 (0.90 \pm 0.04 fold) compared to fetal G55/57 (0.44 \pm 0.04 fold) or neonates NW1 $(0.5 \pm 0.04$ fold). Junctophilin2, a determinant of TT integrity, was expressed in G55/57 and, surprisingly, was invariant among the biological groupings. Analysis of digitally reconstructed (Amira 6.0) serial EM images revealed developmental changes in cardiomyocyte structure. Sarcomere length narrowed from G55/57 $(2.28 \pm 0.01 \mu \mathrm{m})$ to NW1 $(1.90 \pm 0.01 \mu \mathrm{m})$ and adult $(1.92 \pm 0.01 \mu \mathrm{m})$; assessing 250 sarcomeres. Total tubular volume increased from 0.68 $\pm 0.08 \%$ at G64/68 to $2.54 \pm 0.48 \%$ in the adult. Fetal cardiac TTs appear as early as the mid-third trimester of guinea pig pregnancy. Changes in TT/TAT abundance through pre- and post-natal development to adulthood mirror the changes in expression of some (Î́2 adrenoceptor) but not other (junctophilin2) proteins likely to be important for maturation of ECC. TT formation may start prenatally but it is less organised.

\section{LEPTIN INDUCES SCA-1+ PROGENITOR CELL MIGRATION ENHANCING NEOINTIMAL FORMATION}

Yao Xie* . King's College London

\subsection{6/heartjnl-2017-311726.165}

Background Leptin is a hormone that is predominantly secreted by white adipose tissue and thus the majority of obese individuals display high concentrations of plasma leptin. Initially believed to be solely a metabolic factor, leptin also plays a role in inflammation, vascular disease, to which Sca-1 + vascular progenitor cells within the vessel wall may contribute.

Hypothesis Leptin can influence neointimal formation by promoting the migration of Sca-1+ progenitor cells.

Methods and results $\mathrm{Sca}-1+$ progenitor cells were cultivated from the vessel wall of apoE-/- mice and purified via microbeads. The cell migration assessments included transwell and wound healing assays in vitro. The migration of Sca-1+ progenitor cells was markedly induced by leptin in a dosedependent manner $(2.420 \pm 0.222$ and $1.318 \pm 0.036)$. This migration induced by leptin was significantly inhibited by a leptin triple mutant antagonist CYT-566, ERK inhibitor or a STAT3 inhibitor. Western blot analysis revealed that leptin induced phosphorylation of STAT3 and ERK1/2, respectively, implicating the impact of these signal pathways. When applied the Sca-1+ progenitor cells from leptin receptor deficient mice for the experiments above, both the migratory ability and protein activation were markedly abolished. When endovascular injury was induced in wild-type mice by passing a 0.014 guidewire three times through the femoral artery, neointimal lesions were observed in a time-dependent manner. Immunostaining for leptin displayed an increased expression of leptin in the injured vessels. Serum ELISA for leptin assay demonstrated a peak increase at 24 hours after vessel injury. When red fluorescent protein (RFP) labelled-Sca-1+ 